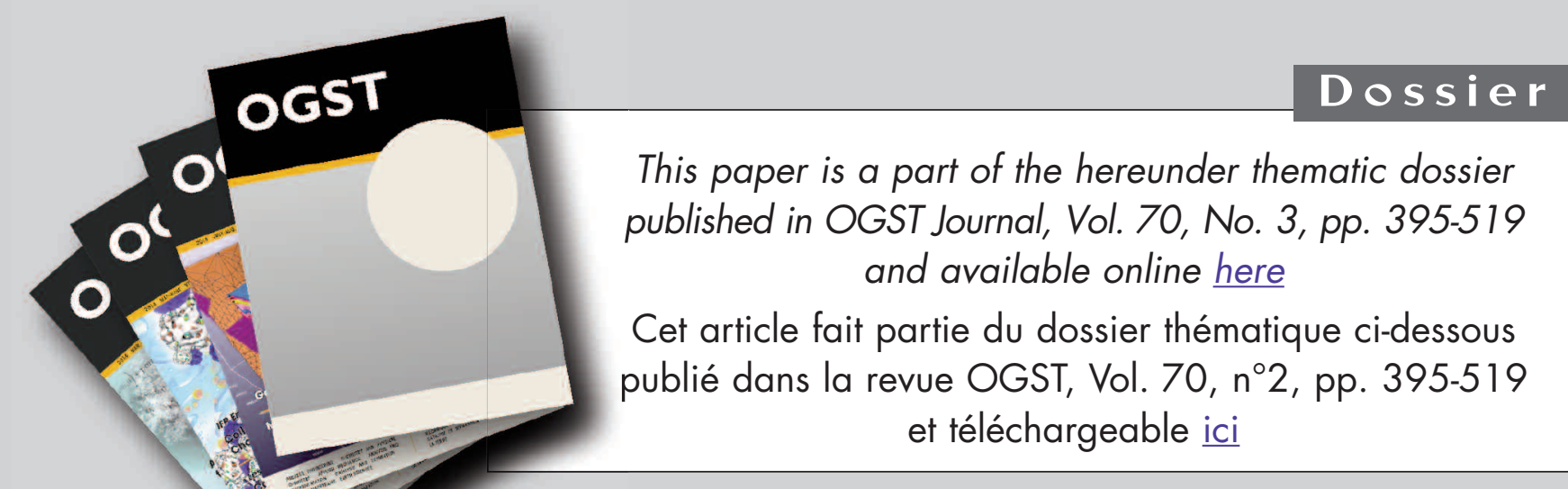

DOSSIER Edited by/Sous la direction de : V. Santos-Moreau

IFP Energies nouvelles International Conference / Les Rencontres Scientifiques d'IFP Energies nouvelles NEXTLAB 2014 - Advances in Innovative Experimental Methodology or Simulation Tools used to Create, Test, Control and Analyse Systems, Materials and Molecules

NEXTLAB 2014 - Innover dans le domaine de la méthodologie expérimentale et des outils de simulation pour créer, tester, contrôler et analyser des systèmes, matériaux et molécules

Oil \& Gas Science and Technology - Rev. IFP Energies nouvelles, Vol. 70 (2015), No. 3, pp. 395-519

Copyright (C) 2015, IFP Energies nouvelles

$395>$ Editorial - Towards the Laboratory of the Future for the Factory of the Future Éditorial - Vers le laboratoire du futur pour construire l'usine du futur V. Santos-Moreau, J.M. Newsam and J.-C. Charpentier

$405>$ Automatic and Systematic Atomistic Simulations in the MedeA ${ }^{\circledR}$ Software Environment: Application to EU-REACH

Simulations atomistiques automatiques et systématiques dans l'environnement logiciel de MedeA® : application à EU-REACH

$X$. Rozanska, P. Ungerer, B. Leblanc, P. Saxe and E. Wimmer

419 > Development of an Innovative XRD-DRIFTS Prototype Allowing Operando Characterizations during Fischer-Tropsch Synthesis over Cobalt-Based Catalysts under Representative Conditions

Développement d'un prototype DRX-DRIFTS innovant permettant des caractérisations operando de catalyseurs à base de cobalt pendant la synthèse de Fischer-Tropsch en conditions représentatives

J. Scalbert, I. Clémençon, C. Legens, F. Diehl, D. Decottignies and S. Maury

429 > Synchrotron X-ray Scattering as a Tool for Characterising Catalysts on Multiple Length Scales

La diffusion des rayons $X$ synchrotron : un outil pour la caractérisation des catalyseurs sur les multiples échelles de longueur

J.M. Hudspeth, K.O. Kvashnina, S.A.J. Kimber and E.P. Mitchell

437 > High Throughput Experimentation (HTE) Directed to the Discovery,

Characterization and Evaluation of Materials

Expérimentation à haut débit pour la découverte, la caractérisation et

l'évaluation des matériaux

J.M. Newsam

447 > The Use of Original Structure-Directing Agents for the Synthesis of EMC-1 Zeolite L'utilisation d'agents structuraux originaux pour la synthèse de zéolithe EMC-1

T.J. Daou, J. Dhainaut, A. Chappaz, N. Bats, B. Harbuzaru, H. Chaumeil, A. Defoin,

L. Rouleau and J. Patarin
455 > REALCAT: A new Platform to Bring Catalysis to the Lightspeed REALCAT : une nouvelle plate-forme pour mener la catalyse à la vitesse de la lumière

S. Paul, S. Heyte, B. Katryniok, C. Garcia-Sancho, P. Maireles-Torres and F. Dumeignil

$463>$ What are the Needs for Process Intensification? Quels besoins pour intensifi er un procédé ?

C. Gourdon, S. Elgue and L. Prat

$475>$ Revisiting the Side Crushing Test Using the Three-Point Bending Test for the Strength Measurement of Catalyst Supports

Test d'écrasement grain à grain revisité à l'aide du test de flexion trois points pour la mesure de la résistance des supports de catalyseurs D. Staub, S. Meille, V. Le Corre, J. Chevalier and L. Rouleau

487 > Refractometric Sensing of Heavy Oils in Fluorescent Core Microcapillaries La détection réfractométrique des huiles lourdes dans les microcapillaires à cœur fluorescents

V. Zamora, Z. Zhang and A. Meldrum

497 > Two-Phase Flow in Pipes: Numerical Improvements and Qualitative Analysis for a Refining Process

Écoulements diphasiques dans les conduites : améliorations numériques et analyse qualitative pour un procédé de raffinage

R.G.D. Teixeira, A.R. Secchi and E.C. Biscaia Jr

511 > Comparative TPR and TPD Studies of Cu and Ca Promotion on Fe-Zn- and Fe-Zn-Zr-Based Fischer-Tropsch Catalysts

Études comparatives par TPR et TPD de la promotion par Cu et Ca de I'activité de catalyseurs Fischer-Tropsch Fe-Zn et Fe-Zn-Zr 0.0 . James, B. Chowdhury and S. Maity 
NEXTLAB 2014 - Advances in Innovative Experimental Methodology or Simulation Tools used to Create, Test, Control and Analyse Systems, Materials and Molecules

NEXTLAB 2014 - Innover dans le domaine de la méthodologie expérimentale et des outils de simulation pour créer, tester, contrôler et analyser des systèmes, matériaux et molécules

\title{
REALCAT: A New Platform to Bring Catalysis to the Lightspeed
}

\author{
Sébastien Paul ${ }^{1,2,3 *}$, Svetlana Heyte ${ }^{1,2,3}$, Benjamin Katryniok ${ }^{1,2,3}$, Cristina Garcia-Sancho ${ }^{4}$, \\ Pedro Maireles-Torres ${ }^{4}$ and Franck Dumeignil ${ }^{1,2,5}$ \\ ${ }^{1}$ Univ. Lille Nord de France, Lille - France \\ ${ }^{2}$ CNRS UMR8 181, Unité de Catalyse et Chimie du Solide, UCCS, Villeneuve d'Ascq - France \\ ${ }^{3}$ École Centrale de Lille, ECLille, 59655 Villeneuve d'Ascq - France \\ ${ }^{4}$ Departamento de Química Inorgánica, Cristalografía y Mineralogía (Unidad Asociada al ICP-CSIC), Facultad de Ciencias, \\ Universidad de Málaga, Campus de Teatinos s/n., 29071 Málaga - Spain \\ ${ }^{5}$ Institut Universitaire de France, Maison des Universités, 103 Bd Saint-Michel, 75005 Paris - France \\ e-mail: sebastien.paul@ec-lille.fr \\ * Corresponding author
}

\begin{abstract}
Catalysis, irrespective of its form can be considered as one of the most important pillars of today's chemical industry. The development of new catalysts with improved performances is therefore a highly strategic issue. However, the a priori theoretical design of the best catalyst for a desired reaction is not yet possible and a time- and money-consuming experimental phase is still needed to develop a new catalyst for a given reaction. The REALCAT platform described in this paper consists in a complete, unique, integrated and top-level high-throughput technologies workflow that allows a significant acceleration of this kind of research. This is illustrated by some preliminary results of optimization of the operating conditions of glycerol dehydration to acrolein over an heteropolyacid-based supported catalyst. It is shown that using REALCAT high-throughput tools a more than 10-fold acceleration of the operating conditions optimization process is obtained.
\end{abstract}

\begin{abstract}
Résumé - REALCAT : une nouvelle plate-forme pour mener la catalyse à la vitesse de la lumière La catalyse, quelle que soit sa forme, peut être considérée comme un des plus importants piliers de l'industrie chimique. Le développement de nouveaux catalyseurs aux propriétés catalytiques améliorées est ainsi d'une importance stratégique capitale. À ce jour la catalyse n'est pas une science prédictive et la conception théorique d'un catalyseur pour une réaction donnée n'est pas encore possible et une approche expérimentale consommatrice en temps et en ressource est nécessaire. La plateforme REALCAT décrite dans cet article consiste en un ensemble complet, intégré et unique au monde d'équipements de criblage permettant une accélération significative de la recherche dans le domaine de la catalyse. Un premier exemple d'optimisation des conditions opératoires de la déshydratation du glycérol en acroléine sur catalyseur hétéropolyanionique est donné à titre d'illustration. Il montre que les outils de la plateforme REALCAT permettent de réduire par au moins un facteur 10 le temps d'optimisation des conditions opératoires de la réaction étudiée.
\end{abstract}




\section{INTRODUCTION AND CONTEXT OF THE PROJECT}

Catalysis, irrespective of its form (homogeneous, heterogeneous or biocatalysis) can be considered as one of the most important pillars of today's chemical industry. It is always present in crucial domains such as Environment, Food, Health, Energy, which are at the inner core of the current societal demands. The catalysts market represented, in $2011,14.7 \mathrm{~T} €$ and it is foreseen to grow up to $17.2 \mathrm{~T} €$ in 2014 and 19.6 $\mathrm{T} €$ in 2016 [1]. The development of new catalysts with improved performances is therefore a highly strategic issue. Moreover, the stakeholders of the market have to take into account the fact that the demand for sustainable processes based on the use of renewable raw materials (i.e., biomass) is stronger and stronger. However, the substrates derived from biomass have most often a very different chemical nature than the compounds issued from the fossil resources. The later are generally hydrophobic and do not contain a lot of oxygen whereas the compounds issued from biomass are mainly hydrophilic and contains many hydroxyl groups or other oxygenated chemical functions. This great difference in the chemical nature of these new raw materials implies that the catalysts used cannot be simply replicated from what is already known by the man of the art, but they rather have to be redesigned to cope with a totally different reactive media than that encountered up to now in petro-chemistry.

\section{METHODS OF INNOVATION IN CATALYSIS}

Irrespective of the form of catalysis, the innovation in this field is based, on the one hand, on a preliminary fundamental research phase devoted to the development of new catalytic concepts and, on the other hand, on a subsequent experimental phase dedicated to the synthesis, to the characterization of the catalysts (analyses of their textural, structural, morphological properties), and on the assessment of their performances. This experimental part is time- and moneyconsuming, as a traditional and iterative "trial and error" method for synthesizing and testing catalysts is still needed. As a matter of fact the a priori theoretical design (i.e., the prediction of the optimal composition, structure and conditions of preparation) of the best catalyst for a desired reaction is not yet possible. Therefore, any mean that could accelerate this experimental phase and hence shorten the development of new catalytic systems is highly desired. That is the goal of High-Throughput Experiments (HTE), which have been actively developed the latest years in the area of catalysis research [2-7].

\section{HISTORY AND KEY DATA OF THE REALCAT PROJECT}

In this context, a first version of what would become later the REALCAT ${ }^{1}$ platform has been imagined in 2010. After some modifications in 2011, the project was accepted in December 2011 and funded by the French Government in the frame of the EQUIPEX part of the 'Future Investments' program (PIA), with the contractual reference 'ANR-11-EQPX-0037'. The global budget represents 8.7 M€ over 10 years (including $6.5 \mathrm{M} €$ of equipment) plus $700 \mathrm{k} €$ from the Nord-Pas-de-Calais Region in the frame of the FEDER program and $10 \mathrm{k} €$ from the Centrale Initiatives Foundation. The French National Research Agency (ANR) is in charge of the administration of the REALCAT project.

The project gathers four laboratories, all located on the same campus in Villeneuve d'Ascq (North of France). UCCS (Unité de Catalyse et de Chimie du Solide, UMR CNRS 8181), the coordinator of the REALCAT program, is already largely recognized for its strong expertise in the fields of heterogeneous and homogeneous catalysis. ProBioGEM ${ }^{2}$ (Procédés Biologiques, Génie Enzymatique et Microbien, EA CNRS 1026) is also a well-known actor of the development of new biocatalytic processes. A strong partnership has also been set with the LAGIS (Laboratoire d'Automatique, Génie Informatique et Signal, UMR CNRS 8219), which is a laboratory specialized in computer engineering and data treatment, and with the LIFL (Laboratoire d'Informatique Fondamentale de Lille, UMR CNRS 8022), a laboratory excelling in informatics, bio-informatics, modelling and analysis of biologic systems, with new recent insights in the synthetic biology ${ }^{3}$. This integrated combination of skills is of outstanding importance for the optimization of the different operations of the platform, and REALCAT will continuously promote high-level R\&D catalysis programs. As far as the human resources are concerned three full-time permanent engineers, from CNRS or Lille University, are in charge of the running and maintenance of REALCAT equipment.

The REALCAT lab, located in the École Centrale de Lille building, has been designed early in 2012. Then, it has been built within the next 12 months to be delivered in the first trimester of 2013. The first equipment was installed before Summer 2013, opening the implementation phase that has ended up before Summer 2014. The REALCAT platform (Fig. 1) is fully operational since the end of 2014, and the exploitation phase is then completely launched.

\footnotetext{
${ }^{1}$ REALCAT is an acronym standing for « RÉacteurs Avancés pour le cribLage en CATalyse appliquée à la valorisation de la biomasse » which could be translated by 'Advanced High-Throughput Technologies Platform for Biorefineries Catalysts Design'.

${ }^{2}$ ProBioGEM will be part of the Charles Viollette Institute in January 2015.

${ }^{3}$ LIFL and LAGIS will merge in January 2015 to create a new lab named CRIStAL.
} 


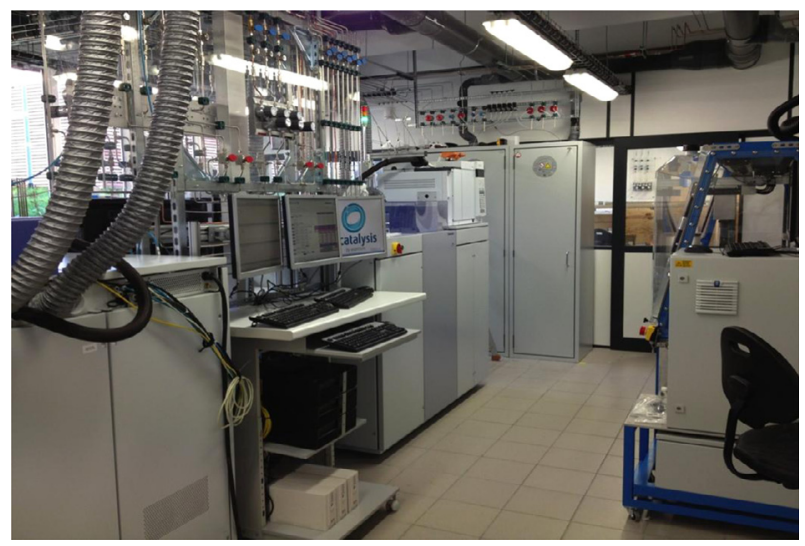

Figure 1

The REALCAT platform in September 2013.

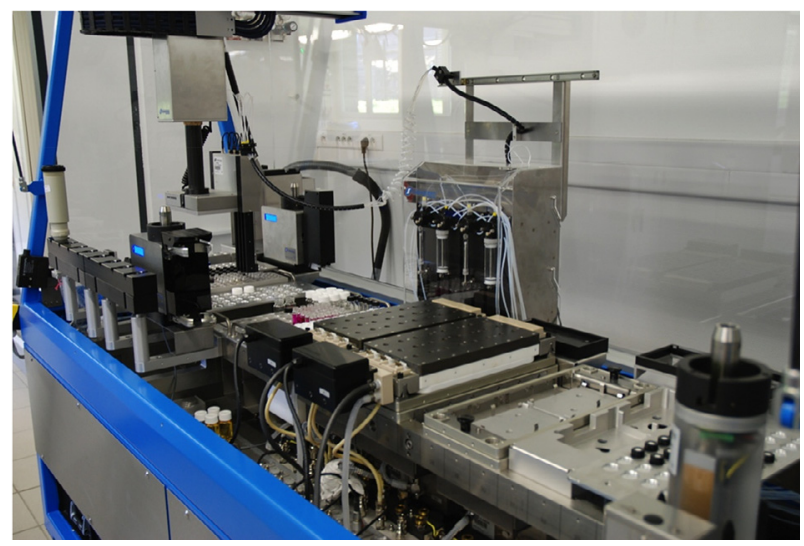

Figure 2

The Chemspeed Catimpreg workstation.

\section{DESCRIPTION OF THE REALCAT PLATFORM}

\subsection{Introduction}

The REALCAT platform consists in a complete, unique, integrated and top-level High-Throughput (HT) technologies workflow including:

- robots for the automated synthesis of catalysts and novel biocatalysts;

- rapid characterization tools;

- a series of versatile parallel continuous and batch reactors and fermentors - for gas phase, liquid phase or multiphase reactions - combined with ultra-fast analytical tools at the top of the state-of-the-art for the analysis of the products in the reactors effluents. All these apparatus are described in details in the following sections.

\subsection{Equipment for the Catalysts Syntheses}

The REALCAT platform comprises two fully automated, flexible and modular robotic workstations supplied by the Swiss company Chemspeed.

The first one, called Catimpreg (Fig. 2), can be used for the automated parallel synthesis of heterogeneous catalysts by co-precipitation or impregnation methods. It handles multistep synthesis procedures such as: reagents preparation, solids and liquids dosing, reaction, filtration, extraction, evaporation, vortexing and reflux. Inert atmosphere for sensitive reagents or inert reaction conditions can be ensured in the Catimpreg workstation either by inertization of the reactors or of the entire workstation enclosed hood. The Catimpreg workstation is equipped with gravimetric and volumetric dispensing tools, used to perform automated and gravimetrically controlled, dispenses of a wide range of solids (powders or extrudates) and liquids in the milligrams to multigrams ranges. A multigripper for vials equipped with a barcode scanner and a screwcapper for opening/closing vials is also present on the workstation. Solid phase extraction cartridges rack allows solid phase extractions. The Catimpreg workstation is also equipped with two reaction blocks designed for the parallel synthesis or reactions. They are consisting of disposable reactors $(24 \times 20 \mathrm{~mL}$ and $12 \times 100 \mathrm{~mL}$, respectively), an array cover plate and an individual shaker unit. The reactors can be used under vacuum, inert gas or without controlled atmosphere. The reactor blocks are equipped with a fluid heating/cooling device and further include a reflux plate. The temperature of the reactors can be controlled in the range of $-10^{\circ} \mathrm{C}$ to $150^{\circ} \mathrm{C}$. A filtration option is also included in the equipment of the reactor blocks.

The second Chemspeed workstation is called Autoplant (Fig. 3). It has been mainly designed for the automated parallel hydrothermal synthesis of catalyst and for liquid phase catalysts testing. Like the Catimpreg workstation, it can perform multi-step synthesis procedures such as: reagent preparation, solids and liquids dosing, reaction, filtration, extraction, evaporation, mixing and reflux. It possesses an $X Y Z$ robotic arm with a rotating alpha-axis and an automatic tool exchange. The Autoplant platform is also equipped with tools for overhead gravimetric dispensing of different types of solids and liquids and volumetric dispensing of liquids. Like for the Catimpreg workstation, inert atmosphere for sensitive reagents or inert reaction conditions can be ensured in the Autoplant workstation either by inertization of the reactors or of the entire workstation enclosed hood. 


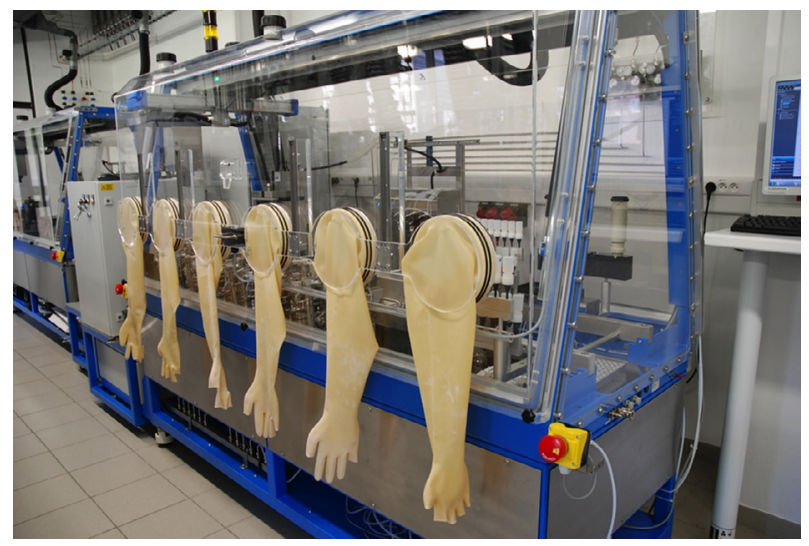

Figure 3

The Chemspeed Autoplant workstation.

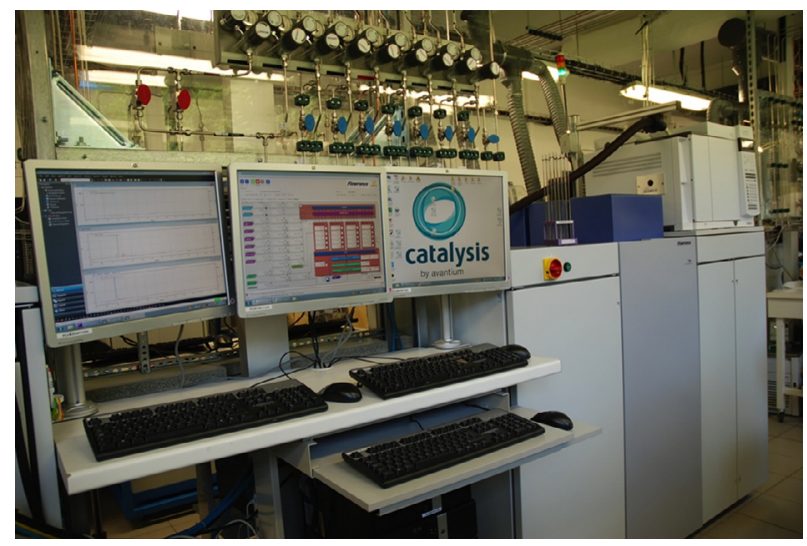

Figure 4

Avantium Flowrence unit.
The hood is equipped with $\mathrm{H}_{2} \mathrm{O}$ and $\mathrm{O}_{2}$ detectors and gloves allowing the user to control the atmosphere inside the workstation and, if necessary, to carry out some tasks manually in the inertizated hood (Fig. 3). Eight fully automated and instrumented high-pressure reactors (autoclaves) are installed in this workstation. Each reactor is equipped with a set of high-pressure pumps, gas flow and pressure controllers. Loading of solid, injection of liquid and gas, pressure (up to 80 bar) and temperature $\left(-10^{\circ} \mathrm{C}\right.$ to $\left.250^{\circ} \mathrm{C}\right)$ can be controlled individually in each reactor. The reactors heads include an integrated reflux condenser and connections for temperature and $\mathrm{pH}$ probes and liquid sampling. Different stirrer designs (anchor or twisted blade) can be used, and are easily exchanged. Indirect online viscosity measurements can be carried out. In addition, reagents can be added during an application via a syringe pump. The reactors can be used in the batch, semi-batch or continuous modes.

No HT thermal treatment unit is available on REALCAT at the moment. So the catalysts' activations are made in classical oven or directly in the reactors where they are tested.

The biocatalysis part of the REALCAT platform is equipped with HT robots that enable the production, purification, characterization and testing of libraries of mutants by directed and random evolutions of enzymes. These tools are not described in details in the present paper, mainly focused on the chemo-catalysis part.

\subsection{Equipment for the Catalysts Characterization}

The equipments installed in the REALCAT platform also bring a complete solution for HT elemental, structural and surface analysis of the catalysts (or of solids in general, and liquids for the adapted techniques). An X-ray fluorescence spectrometer (M4 Tornado, Bruker), and an Inductively Coupled Plasma Optical Emission Spectrometer (ICP-OES 720-ES, Agilent) can be used to perform multi-elemental analysis either of solid or liquid samples. Crystalline phase identification and quantification can be done using an X-ray diffraction spectrometer (D8 Discover, Bruker). Two vibrational spectroscopic techniques, namely the Fourier Transformed Infrared Spectrometry (FTIR) (Tensor 27, Bruker) and the Raman spectroscopy (Xplora, Horiba) can be used in REALCAT. All of these tools, except ICP that uses a specific sampler described hereafter, allow HT analysis of the samples using microplate holders or extensions. The sampling plates for the analysis, compatible with all spectrometers, contain 16 wells. Introduction of liquid samples in the ICP-OES is provided by a HT autosampler (SPS3 Autosampler, Agilent). Samples preparation for ICP analysis is performed in a fully automated digestion Vulcan workstation. These REALCAT platform analysis tools are completed by a set of gas phase and liquid phase chromatographs (see their description below) as well as by HPLC-TOF and MALDI-TOF equipments.

\subsection{Equipment for the Measurement of the Catalytic Performances}

The REALCAT platform is equipped with three compact, stand-alone fixed-bed reactor Flowrence systems supplied by Avantium (Fig. 4). These units were designed to screen heterogeneous catalysts for many different reactions such as Fischer Tropsch, VOC abatement, reforming, $\mathrm{deNO}_{x}$, oxidation, dehydration and so on. The 3 units enable covering a wide range of operating conditions, and, hence, a wide range of reactions can be studied. Each unit contains 4 blocks that 
can be operated at different temperatures. Each block contains 4 stainless steel or quartz reactors, that is to say that 16 isothermal fixed-bed micro flow reactors can be ran in parallel, and that 16 experiments can be conducted simultaneously. Between 50 to $500 \mathrm{mg}$ of catalyst can be loaded in one reactor (powder). As aforementioned, the units can operate in wide operating conditions ranges: pressure can be set between the atmosphere and $90 \mathrm{bar}$, temperature between $50^{\circ} \mathrm{C}$ and $750^{\circ} \mathrm{C}$, feed can be either gaseous, liquid or a mixture of both. All the Flowrence units are equipped with a 16-ports multi position valve that enables online gas phase analysis using a gas chromatograph (Agilent 7890). Two of the three units are equipped with liquid sampling robots that allow collecting the effluent of each reactor separately. The cold traps are maintained in the sampling robot at a temperature ranging between $10-80^{\circ} \mathrm{C}$ using an external cooling/ heating device.

Further, the collected samples can be analysed either by off-line fast gas chromatograph (Shimadzu GC-2010 Plus equipped with two analytical FID channel or Shimadzu GCMS-QP2010 Ultra EI equipped with two analytical channel FID and MS), or by fast high performance liquid chromatograph (Shimadzu equipped with two detectors: LCMS 2020 mass spectrometer simple quadruple and SPD-M20A diode array detector). Each analytical system has been designed to analyse a maximum of 256 samples per day.

The REALCAT platform is also equipped with a multiangle light scattering detector (miniDAWN TREOS, Wyatt) and with a refractometer with extended range (Optilab T-rEX, Wyatt) coupled with an HPLC equipped with an UV spectrometer (Shimadzu). These tools allow determining the absolute molar masses and sizes of polymers or biopolymers.

In operation, more than 128 samples can be collected per day by a single Flowrence unit equipped with a sampling robot. To prepare this huge amount of samples before analysis, a robotic system (Lissy-1 G, Zinsser) is used. This robot is equipped with a liquid handling system, a crimping station, a single vortexer and a cooling/heating device, and thus allows the sample transfer from the Flowrence cold traps to standard $2 \mathrm{~mL} \mathrm{GC}$ vials, dilution, addition of internal standard for off-line analysis and maintaining the sample at the required temperature.

Liquid phase catalyst testing is also possible at ambient pressure using the Chemspeed Catimpreg workstation, equipped with 36 reactors, and at pressure up to 80 bar using the Chemspeed Autoplant workstation, equipped with 8 reactors. These robotic workstations bring a solution to test homogeneous/heterogeneous and enzymatic catalysts in high-throughput conditions.

A set of tools for the testing of biocatalysts is also available on the REALCAT platform. It is not described in details in this article focused on the chemo-catalysis part, as said before.

\section{REALCAT: A PLATFORM OPEN TO EXTERNAL USERS}

\subsection{Introduction}

At full capacity, the platform aims at running 20 projects per year involving worldwide external users from both academic and industrial areas. Among these 20 projects, it is estimated that half of them will be performed in the frame of industrial collaborative projects; $20 \%$ in the frame of academic collaborative projects (including the projects led by the members of the REALCAT consortium only), and $30 \%$ of provisions of services.

A separate office is available close to the REALCAT lab to welcome the external users.

Confidentiality and safety are keywords for the REALCAT project. Important measures have been taken to ensure that all the projects will be conducted in safe conditions for the operators and that the data transmission will be strictly limited to the authorized persons.

\subsection{An Example of a First Academic Collaborative Project}

\subsubsection{Introduction}

In the frame of our collaboration with Prof. Pedro MairelesTorres' team (University of Malaga, Spain), the dehydration of glycerol to acrolein on an heteropolyacid active phase supported on a zirconium-doped SBA-15 mesoporous silica has been studied using some HT tools of the REALCAT platform. Acrolein is of high interest due to its importance as an intermediate for the superabsorbent industry (via acrylic acid) and for feed additives (synthon for $D L$-methionine). It is known that acrolein can be obtained by glycerol catalytic dehydration over acid catalysts, such as zeolites and supported heteropolyacids [8]. The reaction conditions vary according to the employed catalysts, whereby the optimization of the latter is required for each solid independently.

\subsubsection{Methodology}

Today, the most common way to optimize the reaction conditions at the lab scale for a given catalyst is the variation of each parameter one-by-one, meaning that, first, the catalyst amount is optimized, then the reaction temperature is optimized (employing the previously identified optimized catalyst amount), before optimizing the reactant(s) concentration (again using the previously optimized catalyst amount and reaction temperature) and so on. The disadvantage of this sequential approach is the risk to oversee crossinteractions between the reaction parameters. Nevertheless, in order to take these latter into account, for a three level design (high, medium, low) optimizing the aforementioned 
three parameters, $3^{3}$ meaning 27 individual experiments are necessary. Generally, one experiment is done at a time and can take more than 24 hours. This is therefore a very timeconsuming procedure. In order to minimize the number of experiments and still gaining a maximum of information, design of experiments methods based on statistics have been introduced. These latter, combined with a parallel HT testing equipment enabling the simultaneous test of 16 reactors, is a very powerful tool to optimize reaction conditions in a very short time, as it will be shown below.

\subsubsection{Experimental}

A zirconium-doped mesoporous SBA-15 silica was synthesized following the method proposed by Fulvio et al. [9] with some adjustments. First, the structuring agent P123 was solubilized in a $1.7 \mathrm{M}$ hydrochloric acid solution under stirring at $40^{\circ} \mathrm{C}$. Right after, the sources of silica and zirconium (tetraethylorthosilicate and zirconium $n$-propoxide, respectively) were added drop by drop $(\mathrm{Si} / \mathrm{Zr}$ ratio $=5)$. The final molar composition of the synthesis gel was $\mathrm{P} 123 / \mathrm{SiO}_{2} / \mathrm{ZrO}_{2} / \mathrm{HCl} / \mathrm{H}_{2} \mathrm{O}=1 / 55 / 11 / 350 / 11100$. The resulting suspension was stirred at $40^{\circ} \mathrm{C}$ for $72 \mathrm{~h}$, before the solid product was recovered by filtration, washed and dried at $60^{\circ} \mathrm{C}$, and finally calcined in air at $550^{\circ} \mathrm{C}$ for $6 \mathrm{~h}$ with a heating rate of $1 \mathrm{~K} \cdot \mathrm{min}^{-1}$ in order to remove the surfactant molecules. The obtained support was then impregnated with phosphotungstic acid $\left(\mathrm{H}_{3} \mathrm{PW}_{12} \mathrm{O}_{40} \cdot x \mathrm{H}_{2} \mathrm{O}\right)$. The impregnation was performed adding the heteropolyacid $(0.2 \mathrm{~g})$ to a slurry of support $(0.8 \mathrm{~g})$ in water $(10 \mathrm{~mL})$, followed by stirring for $2 \mathrm{~h}$. Then, the water was evaporated under vacuum. The obtained catalyst $(20 \mathrm{wt} \%$ of active phase dispersed on the support) was dried in air for $24 \mathrm{~h}$ at $343^{\circ} \mathrm{C}$ before use.
The catalytic tests were performed in the REALCAT lab using a Flowrence unit equipped with 16 parallel reactors disposed in 4 individually heated blocks. The reactants were fed via a HPLC Jasco pump ( $240 \mathrm{NmL} / \mathrm{min}$ for the glycerol aqueous solution) and a Bronkhorst Mass Flowmeter (32 NmL/min for He) integrated in the Flowrence unit and split equally between the 16 reactors by a heated capillary system, whereby the evaporation of the glycerol solution took place inside the reactor. The pressure was maintained constant at $1.2 \mathrm{~atm}$ in all the reactors. The liquid products were condensed at $30^{\circ} \mathrm{C}$ during $150 \mathrm{~min}$ and then analyzed by gas chromatography. The gases were analyzed by an online GC equipped with TCD and FID detectors (Agilent 7890A). The products collected in the cold traps were analyzed by offline GC (Shimadzu GCMS-QP2010 Ultra EI) equipped with two analytical channels FID and MS.

The parameter optimization was performed with the help of a computer-assisted experimental design (DesignExpert $5^{\circledR}$; Box Behnken response surface) in which 17 experiments were performed. Three parameters were optimized, namely: the amount of catalyst $(50,125$ and $300 \mathrm{mg})$; the reaction temperature $\left(275,300\right.$ and $\left.325^{\circ} \mathrm{C}\right)$ and the glycerol concentration (10, 20 and $30 \mathrm{wt} \%$ in water). The results were fitted employing a quadratic mathematical model.

\subsubsection{Results and Discussion}

From the results, one can see that, for a constant glycerol concentration of $20 \mathrm{wt} \%$ in the feed, the model foresee a conversion increase - as expected - with the reaction temperature and in the lower range of temperature with the amount of catalyst (Fig. 5a). On the other hand,

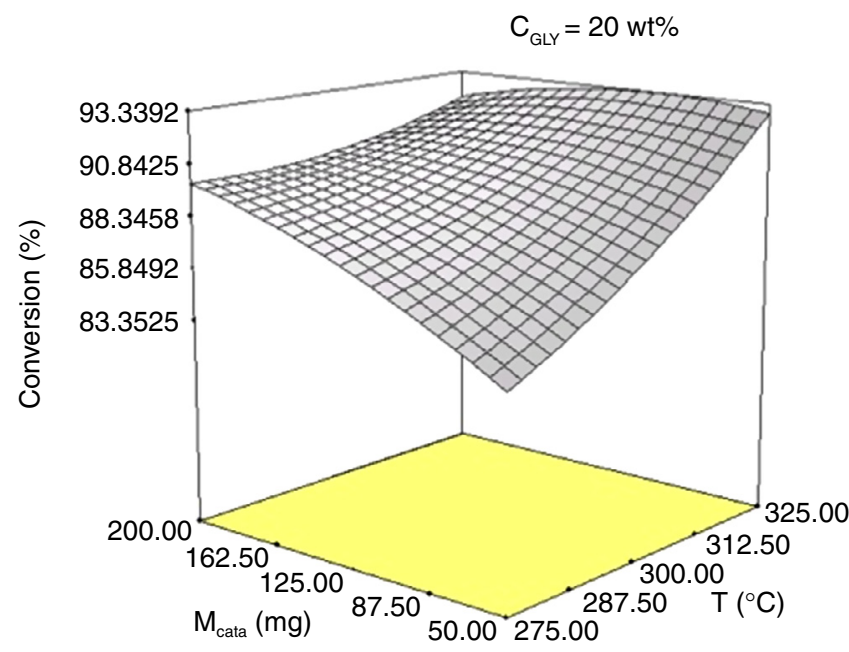

a)

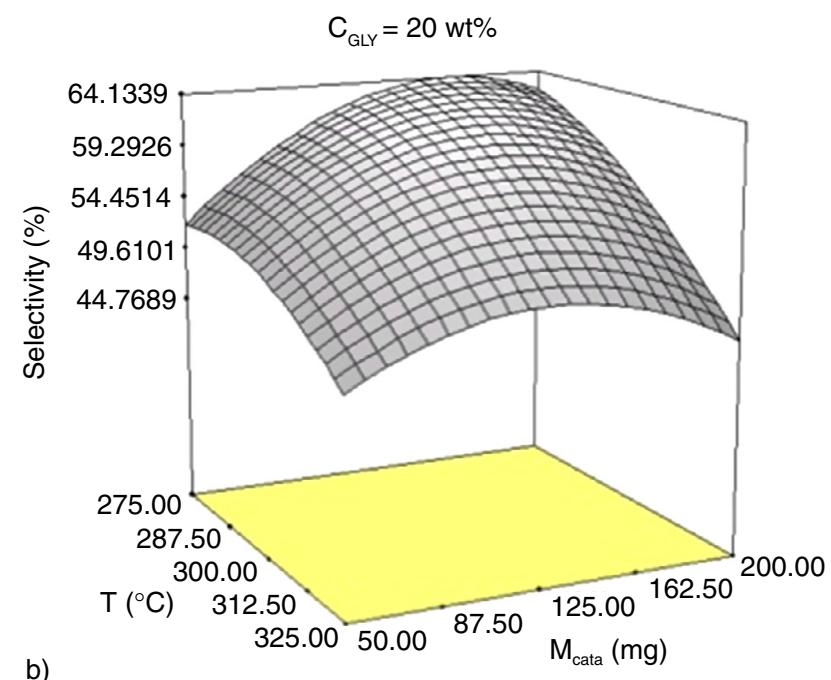

b)

Figure 5

Conversion and selectivity predicted for $20 \mathrm{wt} \% \mathrm{H}_{3} \mathrm{PW}_{12} \mathrm{O}_{40}$ on $\mathrm{ZrO}_{2}-\mathrm{SiO}_{2}$ at constant glycerol feed concentration (20 wt $\%$ ). 

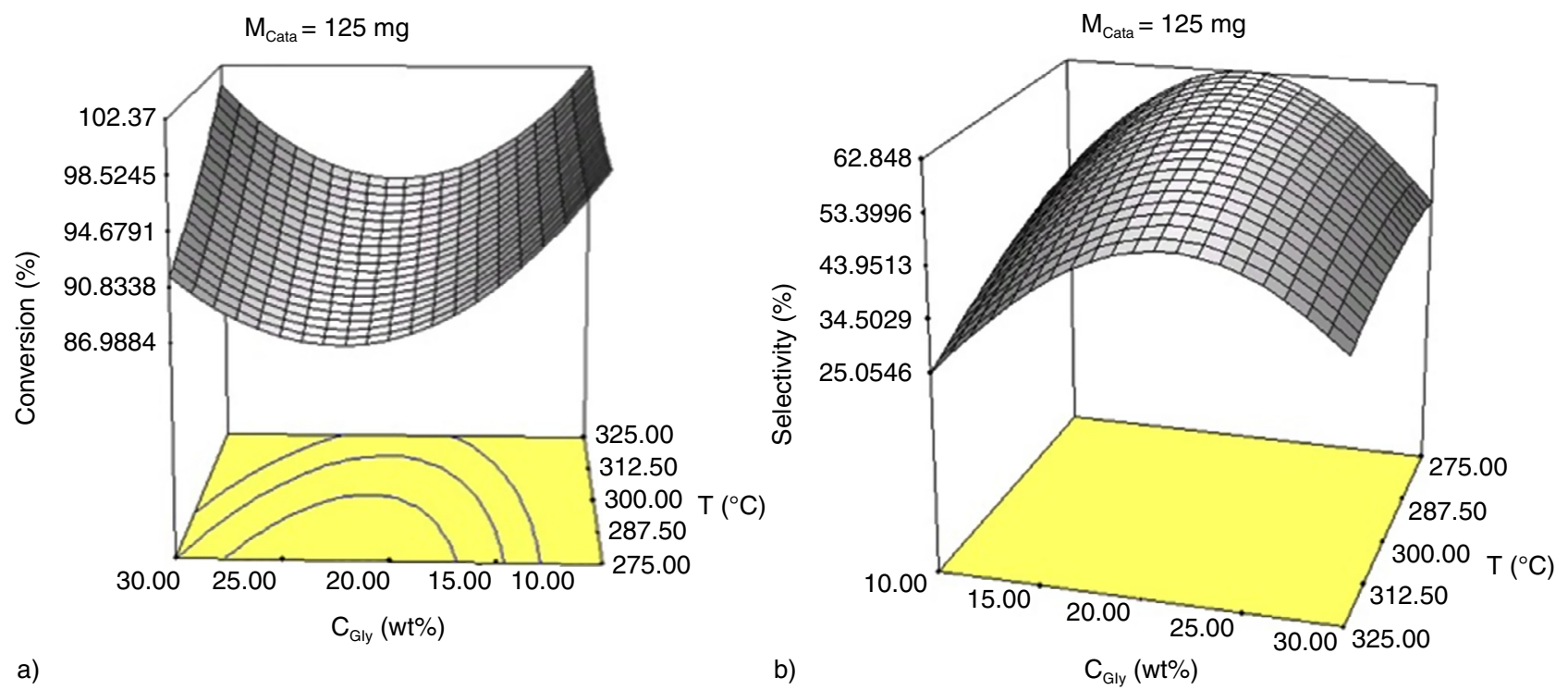

a) b)

$$
\mathrm{C}_{\text {Gly }}(\mathrm{wt} \%)
$$

Figure 6

Conversion and selectivity predicted for $20 \mathrm{wt} \% \mathrm{H}_{3} \mathrm{PW}_{12} \mathrm{O}_{40}$ on $\mathrm{ZrO}_{2}-\mathrm{SiO}_{2}$ at constant mass of catalyst (125 mg).

the selectivity to acrolein (Fig. 5b) significantly dropped for increased reaction temperature (i.e., $45 \%$ at $325^{\circ} \mathrm{C} v s 64 \%$ at $275^{\circ} \mathrm{C}$ ) and showed an optimum for a catalyst amount of $125 \mathrm{mg}$.

Concerning the influence of the glycerol concentration, one can see from Figure 6a that the conversion increased for high (30 wt \%) and low (10 wt\%) concentrations in glycerol. On the other hand, the selectivity (Fig. 6b) showed the reverse trend, since it was the highest $(64 \%)$ for a medium concentration in glycerol $(20 \mathrm{wt} \%)$. This result can be explained by the formation of acetals from glycerol and acrolein as proven by GC-MS analysis (not shown here) [10]. In fact, at a high concentration in glycerol (30 wt\%), a part of the latter is not catalytically converted. Thus, this non-reacted glycerol can form an acetal with acrolein, whereby a part of the catalytically formed acrolein is consumed. Correspondingly, the observed conversion is increased and the selectivity to acrolein decreased.

These preliminary results show that the combination of an experimental design and HT parallel testing equipment enabled rapid and time efficient evaluation of catalytic performance. The results reported in this paper correspond to a total number of 17 individual experiments as said above. They were obtained on the REALCAT platform in less than two days. Such a data collection would have taken nearly one month in a classical catalytic set-up. This represents at least a 10 -fold decrease of the duration of this kind of study.

\section{CONCLUSIONS}

The last two years the REALCAT platform has been designed and set up. It is today a "unique in the world" tremendously powerful operational tool for the design and optimization of new catalysts. The uniqueness of REALCAT is mainly due to its different levels of integration: all kinds of catalysis can be treated (homogeneous, heterogeneous, biocatalysis and even hybrid catalysis), HT tools for the synthesis, the characterization and the testing of catalysts are available and the team leading the project presents multidisciplinary skills (chemo-catalysis, biocatalysis, computer engineering, statistics, etc.). A more than 10 -fold acceleration of the research projects is already obtained, as it has been illustrated in this paper. However, we are just at the beginning of the REALCAT adventure, and a lot of work has still to be done. For instance, the treatment of the huge amount of data generated by the HT instruments will be a challenge. No doubt that the input of the LIFL and LAGIS labs in this domain will be very helpful. In the same area, the set-up of a Laboratory Integrated Management System (LIMS) in the next months, including database gathering the information issued from a given sample from all the instruments of the platform, will surely strongly reinforce the possibilities of interpretation of the data collected on REALCAT.

Within the frame of the REALCAT project, methodological developments will also be carried out in partnership with 
private companies in order to develop innovative HT tools for catalysis not yet available on the market. This will ensure that REALCAT remains the most advanced HT platform for catalysis in the world in the future.

This will open the gate to the development of hybrid catalysts combining enzymatic and chemical active sites in the same catalytic reactor. In that frame the competencies of the researchers of ProBioGEM will be very important. REALCAT will for sure bring to the lightspeed a lot of catalytic processes in the near future.

\section{ACKNOWLEDGMENTS}

The REALCAT platform is benefiting from a Governmental subvention administrated by the French National Research Agency (ANR) within the frame of the 'Future Investments' program (PIA), with the contractual reference 'ANR-11EQPX-0037'. The Nord-Pas-de-Calais Region and the FEDER as well as the Centrale Initiatives Foundation are thanked for their financial contribution to the acquisition of the equipment of the platform. The Universite Lille 1 Sciences \& Technologies (USTL) is also acknowledged for having financed a part of the consulting fees for designing the program and the École Centrale de Lille (ECLille) is warmly thanks for their financial contribution necessary for the setup of the implantation laboratories. The consulting was entrusted to the ALMA Consulting Group, and we would like to warmly thank Dr. Kosta Skarvelakis and Dr. Jetta Keranen for their professionalism and for having given us precious advices for making the project proposal a success, thanks to their deep expertise. Further, we want also to thank the main participants of this program, who actively helped in designing the project, namely Prof. Pascal Dhulster, Dr. Rénato Froidevaux, Prof. Philippe Jacques, Prof. Philippe Vanheeghe, Prof. Emmanuel Duflos, Prof. Sophie Tison and Dr. Cédric Lhoussaine, and who are now actively working on the development of the platform. All the members of the 4 involved laboratories (UCCS, ProBioGEM, LIFL and LAGIS) are also warmly acknowledged for making this platform living through projects in which they bring their strong and precious scientific expertise.

\section{REFERENCES}

1 Data available at http://www.freedoniagroup.com/WorldCatalysts.html.

2 Bouchy C., Duchene P., Faraj A. (2013) Using High Throughput Experimentation Approach for the Evaluation of Dehydrogenation Catalysts: Potential Interests and Drawbacks, Oil Gas Science Technology - Rev. IFP Energies nouvelles 68, 3, 429-443.

3 Morra G., Farrusseng D., Bouchy C., Morin S. (2013) Knowledge Based Catalyst Design by High Throughput Screening of Model Reactions and Statistical Modelling, Oil Gas Science Technology - Rev. IFP Energies nouvelles 68, 3, 487-504.

4 Jolly J., Pavageau B., Tatibouet J.M. (2013) High Throughput Approach Applied to VOC Oxidation at Low Temperature, Oil Gas Science Technology - Rev. IFP Energies nouvelles 68, 3, 505-517.

5 de Vries J.G., Lefort L. (2013) Development of Asymmetric Hydrogenation Catalysts via High Throughput Experimentation, Oil Gas Science Technology - Rev. IFP Energies nouvelles 68, 3, 519-528.

6 Beltran-Oviedo T.A., Batyrshin I., Dominguez J.M. (2009) The optimal design of experiments (ODOE) as an alternative method for catalysts libraries optimization, Catal. Today $\mathbf{1 4 8 ,}$ 28-35.

7 Farrusseng D. (2008) High-throughput heterogeneous catalysis, Surf. Sci. Rep. 63, 487-513.

8 Katryniok B., Paul S., Dumeignil F. (2013) Recent developments in the field of catalytic dehydration of glycerol to acrolein, ACS Catalysis 3, 1819.

9 Fulvio P.F., Pikus S., Jaroniec M. (2005) Tailoring properties of SBA-15 materials by controlling conditions of hydrothermal synthesis, J. Mat. Chem. 15, 5049-5053.

10 Deleplanque J., Dubois J.-L., Devaux J.-F., Ueda W. (2010) Production of acrolein and acrylic acid through dehydration and oxydehydration of glycerol with mixed oxide catalysts, Catal. Today 157, 351.

Manuscript submitted in May 2014 Manuscript accepted in November 2014 Published online in February 2015 